\title{
Alargamiento de corona estético previo a rehabilitación protésica. Informe de caso
}

\section{Aesthetic crown lengthening prior to prosthetic rehabilitation. Case report.}

\begin{abstract}
Alejandra Alvarado-Núñez ${ }^{1}$, Sonora Ramírez-Duarte ${ }^{2}$, Alejandro Nieto-Ramírez $^{3}$, René García-Contreras ${ }^{1 *}$
1. Escuela Nacional de Estudios Superiores, unidad León, UNAM. León, México

2. Escuela Nacional de Estudios Superiores, unidad León, UNAM. León, México

3. Universidad Nacional de Estudios Superiores, unidad León. León, México

* Corresponding Author: René García-Contreras Escuela Nacional de Estudios Superiores (ENES) Unidad León, UNAM. Blvd. UNAM 2011 Predio El Sauce y el Potrero Comunidad Tepetates C.P.37684 León, Gto. 7228856878 | E-mail: dentist.garcia@gmail.com

Trabajo recibido el 06/08/2017

\section{RESUMEN}

Introducción: Uno de los procedimientos realizados con mayor frecuencia para el restablecimiento de la estética en la zona anterosuperior es el alargamiento de corona, el cual está indicado ante la presencia de una longitud insuficiente de la corona clínica de un diente, cuando existe caries o fracturas subgingivales y para la mejora de la estética en pacientes con margen gingival desigual. Objetivo: El objetivo de este artículo es presentar el alargamiento de corona estético realizado en una paciente que presentaba márgenes discrepantes y pérdida de las papilas en la zona anterosuperior, la cual fue remitida del área de prótesis para poder realizar posterior rehabilitación protésica. Resultados: Mediante el procedimiento quirúrgico se logró la corrección y restablecimiento de los márgenes gingivales. Conclusiones: La zona anterosuperior es el área estéticamente más comprometida, por lo que es indispensable la planeación interdisciplinaria del tratamiento para asegurar el éxito del mismo.
\end{abstract}

Aprobado para su publicación el 07/12/2017

PALABRAS CLAVE
Alargamiento de corona; Osteotomía; Estética dental; Gingivoplastía.

Rev. Clin. Periodoncia Implantol. Rehabil. Oral Vol. 11(3); 170-172, 2018.

\section{ABSTRACT}

Introduction: One of the most frequently performed procedures for aesthetics restoration in the maxillary anterior area is the crown lengthening, which is indicated in the presence of an insufficient length of the clinical crown of a tooth, of cavities or subgingival fractures and for the improvement of aesthetics in patients with uneven gingival margin. Objective: The aim of this article is to present the aesthetic crown lengthening performed in a patient with discrepant margins and loss of the papillae in the maxillary anterior area, referred from the prosthesis department for subsequent prosthetic rehabilitation. Results: Through the surgical procedure, the correction and restoration of the gingival margins were achieved. Conclusions: The maxillary anterior area is the most aesthetically compromised area, which is why the interdisciplinary planning of the treatment is essential to ensure its success.

\section{KEY WORDS}

Crown lengthening; Osteotomy; Dental esthetics; Gingivoplasty.

Rev. Clin. Periodoncia Implantol. Rehabil. Oral Vol. 11(3); 170-172, 2018.

\section{INTRODUCCIÓN}

El conocimiento de la morfología dental y gingival normal es esencial para comprender el marco de la sonrisa y lograr una rehabilitación exitosa y predecible de la misma ${ }^{(1)}$. Existen diversos procedimientos mediante los cuales se puede lograr obtener una sonrisa estética, siendo el alargamiento de corona uno de los realizados con mayor frecuencia en la periodoncia. El alargamiento de corona, es un procedimiento quirúrgico que tiene como objetivo exponer la estructura del diente sano mediante el reposicionamiento apical del tejido gingival con o sin extracción del hueso alveolar ${ }^{(2)}$. Dicho procedimiento puede realizarse tanto por fines funcionales como estéticos, especialmente en el sector anterior en el caso de los últimos. Comúnmente, está indicado en la presencia de una longitud insuficiente de la corona clínica de un diente, cuando existe caries o fracturas subgingivales con posibilidad de restauración y para la mejora de la estética en pacientes con margen gingival desigual y exposición excesiva de la encía ${ }^{(3)}$. Existen varios aspectos que se deben considerar antes de realizar un alargamiento de corona, un periodonto sano es un prerrequisito para controlar la respuesta de los tejidos gingivales ${ }^{(4)}$ y la interconsulta con el rehabilitador es necesaria para determinar el tipo de restauración a realizar, lo cual permite evaluar la viabilidad para ejecutar el procedimiento quirúrgico. Se debe tomar en cuenta la posición del diente en la arcada, valor estratégico del mismo, el ancho de la encía queratinizada, la distancia desde la unión cemento-esmalte a la cresta alveolar alrededor de los dientes implicados, relación corona-raíz, anatomía de la raíz, biotipo gingival y grosor del hueso alveolar(1), así como se debe realizar un estudio de la sonrisa del paciente al tratarse la zona anterosuperior. Uno de los objetivos principales al realizar el alargamiento de corona es el mantenimiento del ancho biológico, el cual es utilizado ampliamente como una guía clínica durante la evaluación de interrelaciones reparadoras periodontales ${ }^{(5)}$. Gargiulo et al lo definió como la medida comprometida desde el fondo del surco gingival a la cresta alveolar, abarcando desde el epitelio de unión $(0,97 \mathrm{~mm})$ hasta la inserción del tejido conectivo $(1,07 \mathrm{~mm})$, dando un promedio de $2.04 \mathrm{~mm} \cdot{ }^{(6)}$ Ingber et al sugirieron que $1 \mathrm{~mm}$ adicional debería ser añadido para establecer la distancia óptima entre la cresta ósea y el margen restaurador para asegurar una correcta cicatrización del periodonto ${ }^{(7)}$. Cuando el ancho biológico es invadido, como mecanismo de defensa, la respuesta inflamatoria acelera la pérdida ósea para proporcionar espacio para el nuevo tejido conectivo, que da como resultado 
una recesión gingival o pérdida de la inserción ${ }^{(6)}$. La valoración de la cresta alveolar es otro factor importante, ya que el grado de elongación clínica de la corona con respecto a la posición del hueso alveolar determinará la viabilidad del procedimiento, los aspectos quirúrgicos y la secuencia de tratamiento ${ }^{(5)}$ El tiempo que deberá pasar para que la rehabilitación protésica pueda ser llevada a cabo posterior al procedimiento quirúrgico también es un factor importante, Hempton et al después de una revisión de diferentes autores llegó a la conclusión de que el periodo de cicatrización ideal antes de colocar la restauración final es de 6 meses ${ }^{(8)}$. El objetivo del presente reporte de caso es reportar los resultados obtenidos mediante la técnica quirúrgica de elevación de colgajo de espesor total en una paciente que necesitaba alargamiento de corona para una posterior rehabilitación protésica.

\section{INFORME DEL CASO}

Paciente femenino de 38 años de edad, ASA I, aparentemente sana, sin antecedentes personales de relevancia y hábito de tabaquismo y toxicomanías negado, llegó en mayo del 2016 a la clínica de prótesis de la Escuela Nacional de Estudios Superiores unidad León de la UNAM con motivo de consulta "quiero arreglarme los dientes de adelante". La paciente fue valorada protésicamente y se remitió al área de periodoncia para valoración periodontal, donde se obtuvo como diagnóstico periodontitis crónica generalizada moderada. Después de pasar por la fase I periodontal (eliminación de cálculo, así como raspado y alisado radicular) y de tener a la paciente en fase de mantenimiento (Figura 1), se valoró para alargamiento de corona estético que abarcara del segundo premolar superior derecho al segundo premolar superior izquierdo (15-25) para posterior rehabilitación protésica. Radiográficamente se observó una porción corona-raíz y altura adecuada de hueso alveolar (Figura $2 a$ y $2 b$ ). A la inspección clínica se observó diastema en los centrales superiores, pérdida de papilas, discrepancia de los márgenes gingivales en la zona anterosuperior y un biotipo gingival grueso (Figura 3a), al igual que una línea de sonrisa alta (Figura $3 b$ ). Según las condiciones de la paciente y las consideraciones para realizar un alargamiento de corona, se decidió que el procedimiento era viable. El área de prótesis realizó la guía quirúrgica (mock up) según el encerado diagnóstico y análisis de sonrisa digital diseñado.

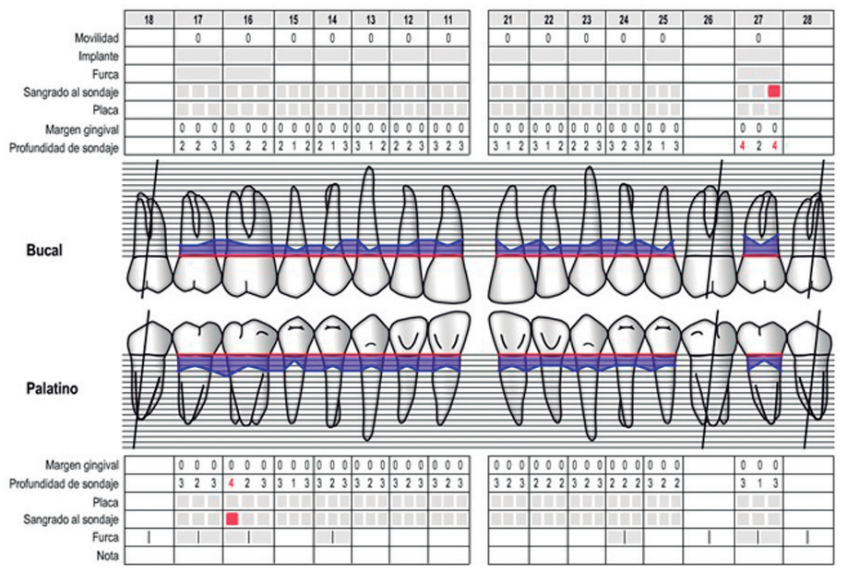

Figura 1. Ficha periodontal de mantenimiento.

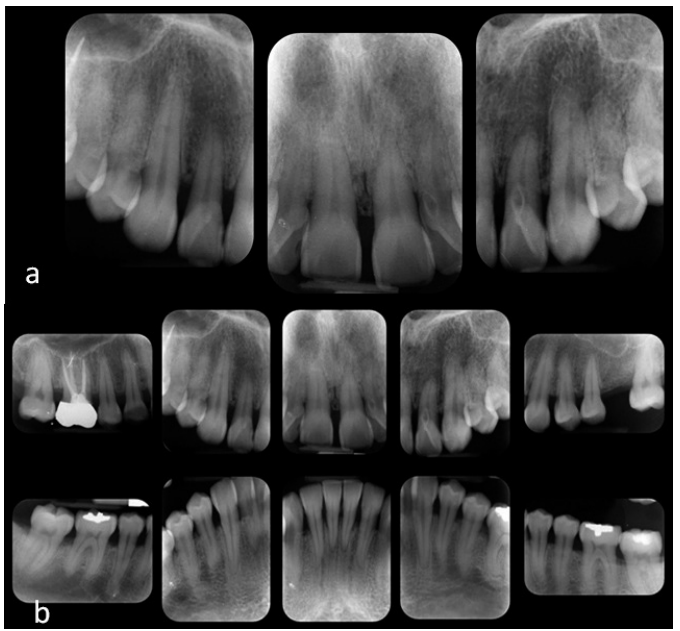

Ficha 2. 2a. Radiografías periapicales de la zona quirúrgica. 2b. Serie radiográfica.

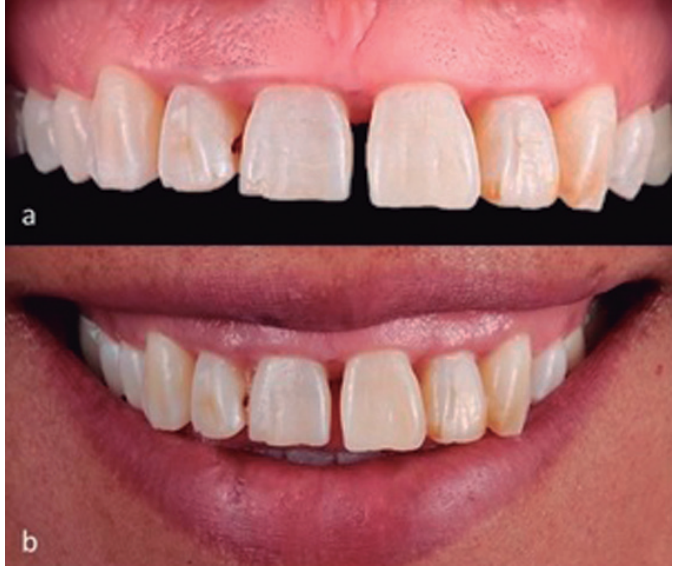

Figura 3. Características clínicas iniciales 2a. diastema en los centrales superiores, pérdida de papilas, discrepancia de los márgenes gingivales en la zona anterosuperior y un biotipo gingival mediana, $2 \mathrm{~b}$. línea de la sonrisa alta.

\section{Procedimiento quirúrgico}

El 25 de agosto de 2016, día en que se realizó el procedimiento, la paciente firmó el consentimiento informado, declarando estar de acuerdo en la utilización de su caso para fines académicos. Se inició el alargamiento de corona con la anestesia del segundo premolar superior derecho al segundo premolar superior izquierdo con mepivacaína al $2 \%$ con epinefrina $(1: 100,000)$ (Septodont, México). Se colocó mock up proporcionado por el área de prótesis y se realizaron incisiones con hoja de bisturí 15C (Ribbel, Nueva Delhi) en base al mismo, el cual fue utilizado como guía para delimitar la zona a tratar (Figura 4a). Una vez delimitada la zona, se realizaron incisiones a bisel interno, haciendo los cortes de mesial a distal de cada diente. Enseguida se realizaron incisiones intrasurcales para obtener una banda de tejido gingival, que posteriormente sería retirada con curetas Gracey (Figura 4b). Posterior a esto, con legra Prichard se levantó colgajo de espesor total con el cual se obtuvo acceso al hueso alveolar, donde se pudo observar los márgenes discrepantes (Figura 4c). Se realizó ostectomía seguido de osteoplastía con pieza de mano de baja velocidad con fresa de bola de carburo \#4, usando simultáneamente abundante irrigación con suero fisiológico y respetando las raíces (Figura 4d). Se midió la distancia desde el margen protésico hasta la nueva altura de cresta ósea para obtener una distancia de $3 \mathrm{~mm}$. El hueso remanente en las raíces de los dientes se eliminó con cincel CTG-0 (Figura 4e). Una vez terminado, se suturó el colgajo con ácido poliglicólico 4-0 con puntos suspensorios (Atramat, México) (Figura 4f). Se le recetó a la paciente ibuprofeno de $600 \mathrm{mg}$ uno cada 8 horas durante 3 días y enjuagues con gluconato de clorhexidina al $0.12 \%$ cada 12 horas por 15 días. Se le indicó mantener buena higiene, sin cepillar o hacer uso de aditamentos interproximales en la zona tratada.

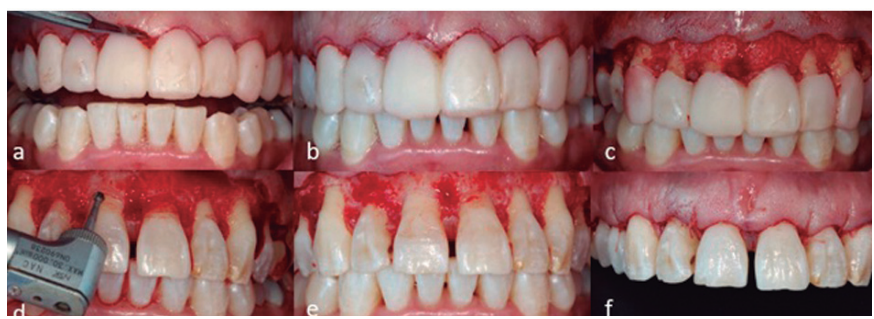

Figura 4. Procedimiento quirúrgico. 4a. Realización de incisiones según el mock-up. 4b. Incisiones a bisel interno 4c. Levantamiento de colgajo 4d. Ostectomía y osteoplastia 4e. Eliminación de hueso remanente con cincel 4f. Colocación de sutura.

\section{Postquirúrgico}

A los siete días (1 septiembre) se realizó retiro de suturas, se observó clínicamente la zona tratada desinflamada, del mismo color de la mucosa adyacente y sin signos de infección (Figura $5 a$ ). A las tres semanas de la cirugía se prepararon los dientes y se colocaron los provisionales (Figura $5 b)$ y a las cinco semanas (30 septiembre) se realizó un recontorneo gingival con ayuda de electrobisturí (Figura 6a). A las nueve semanas se observó una adecuada cicatrización y se procedió a hacer toma de impresión (Figura 6b). El 2 de febrero se colocó la restauración final, observando tejidos sanos y estéticos (Figura 7). Mediante el procedimiento quirúrgico se logró la corrección y restablecimiento de los márgenes gingivales según los requerimientos protésicos, se obtuvo además la longitud coronaria deseada en la planeación del diseño de sonrisa. La realización de recontorneo gingival con electrobisturí 


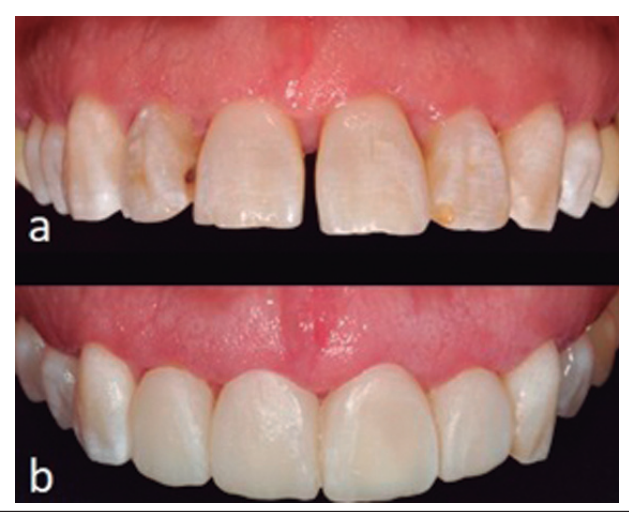

Figura 5. Postoperatorio. 5a Remoción de suturas y vista posquirúrgica a la semana. 5b Colocación de provisionales.

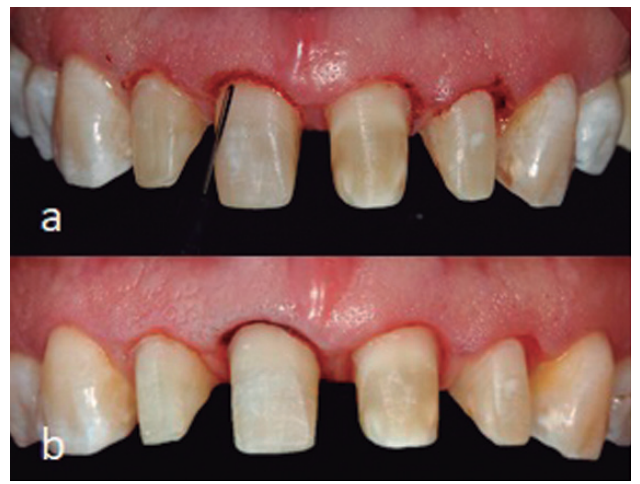

Figura 6. a. Recontorneo gingival con electrobisturí b. Postquirúrgico a las 9 semanas.

permitió crear cénits más armoniosos y ayudó a crear mayor simetría en los márgenes gingivales. La paciente expresó sentirse contenta con los resultados finales tanto de la cirugía como de la colocación de las carillas.

\section{DISCUSIÓN}

Diferentes técnicas quirúrgicas han sido descritas para realizar el alargamiento de corona, una de ellas es la gingivectomía, en la que sólo se elimina el tejido de las superficies vestibulares y se deja la papila interdental intacta, es necesario tener una banda de encía queratinizada lo suficientemente ancha para mantener el ancho biológico una vez realizada la cirugía ${ }^{(9)}$. Otra técnica es mediante un colgajo de reposición apical y este puede ser con y sin reducción ósea ${ }^{(10)}$. Este procedimiento se realiza mediante una elevación de colgajo mucoperióstico, en el cual se expone el hueso alveolar para poder realizar la osteotomía/osteoplastía. En la técnica "flapless", la osteotomía se realiza sin necesidad de realizar la elevación de colgajo, a través del surco gingival utilizando microcinceles o con aparatos piezoeléctricos o escariador. Esta técnica ofrece como beneficios como la ausencia de suturas, menor hemorragia y menor tiempo de cicatrización del surco (3 meses aproximadamente)(11). También se puede realizar el alargamiento de corona mediante una extrusión quirúrgica en la que es muy sencillo conseguir tejido dentario supracrestal sin la necesidad de eliminar tejido de soporte. Consiste en una avulsión del diente sin dañar las tablas óseas y una vez

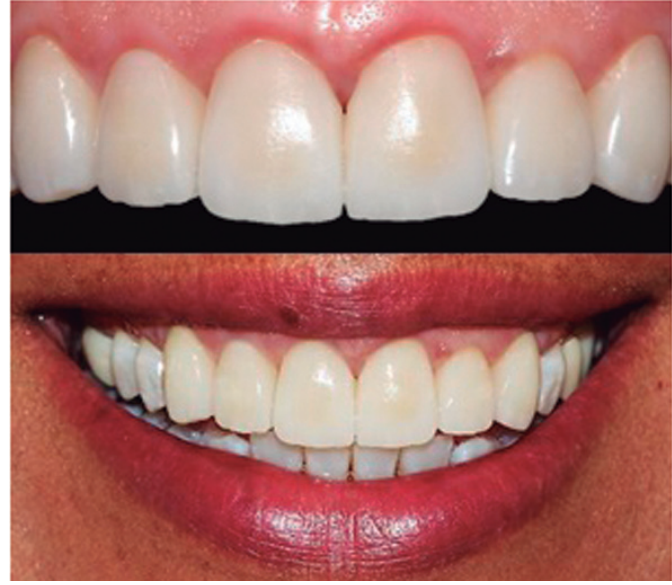

Figura 7. Características clínicas finales.

luxado se posiciona coronalmente dentro del alveolo en la posición idónea, fijándolo a este nivel a través de una ferulización a los dientes adyacentes o con una sutura interdental ${ }^{(9)}$. Nethravathy et al en un estudio realizado a 15 pacientes a los que se les realizó alargamiento de corona mediante gingivectomía, reposición apical y extrusión quirúrgica, llegó a la conclusión tras una evaluación de tres meses de que la extrusión quirúrgica ofrece varias ventajas sobre las otras técnicas, ya que existe una preservación de la papila interproximal, posición del margen gingival y no hubo pérdida ósea ${ }^{(12)}$. Otro método es a través de una erupción forzada con ortodoncia, la cual consiste en la reposición dental mediante movimientos ortodóncicos en dirección coronal aplicando una fuerza continua y suave produciendo cambios en los tejidos blandos y hueso de alrededor ${ }^{(13)}$. La elección de la técnica quirúrgica depende del criterio del clínico y dependerá de las características que presenta cada paciente. En el presente caso, se le informó a la paciente de las diferentes técnicas que podrían realizarse para llevar a cabo su tratamiento, como el uso de ortodoncia, sin embargo, por conveniencia económica y de tiempo, se decidió optar por tratamiento previamente mostrado.

\section{CONCLUSIONES}

La planeación interdisciplinaria de un tratamiento dental es fundamental para asegurar el éxito del mismo. La zona anterosuperior es el área estéticamente más comprometida, por lo que es importante realizar un análisis de sonrisa para determinar las características que llevará la prótesis y en base a esto, realizar la planeación del tratamiento periodontal. Gran parte del éxito del tratamiento a largo plazo radica en el seguimiento que se le haga al paciente, se le debe hacer énfasis en que posterior a la intervención quirúrgica, se debe seguir con el tratamiento periodontal de mantenimiento y revaloración.

\section{CONFLICTO DE INTERÉS}

Los autores declaran no tener conflictos de interés en la publicación del presente artículo

\section{AGRADECIMIENTO}

Los autores agradecen a Eduardo Dorado Suárez por su cooperación en el desarrollo del caso clínico y elaboración del tratamiento protésico.

\section{Bibliografía}

1. Zeina A.K. Majzoub, Romanos A, Cordioli G. Crown lengthening procedures: A literature review. Semin Orthod. 2014;20 (3):188-207.

2. Glossary of periodontal terms, the American Academy of Periodontology, Chicago. Actualizado 10 junio 2012 [citado 31 mayo 2017]. Disponible en: http:// members. perio.org/libraries/glossary/entry?GlossaryKey=3d42cce1-57b0-4c4d8d66-f2eba661923b\&tab=groupdetails.

3. Tomar N, Bansal T, Bhandari M, Sharma A. The perio-esthetic-restorative approach for anterior rehabilitation. J Indian Soc Periodontol. 2013;17:535-8.

4. Martínez HR, Marín Ma.G. Periodontal plastic surgery performed in clinically short crowns for prosthetic rehabilitation Rev Odont Mex. 2013;17(4):236-242.

5 . Lee, E. Aesthethic crown lengthening: contemporary guidelines for achieving ideal gingival architecture and stability. Curr Oral Health Rep. 2017;4:105-111.

6. Gargiulo AW, Wentz FM, Orban B. Dimensions and relations of the dentogingival junction in humans. J Periodontol. 1961;32:261-267.

7. Ingber JS, Rose LF, Coslet JG. The "biologic width": a concept in periodontics and restorative dentistry. Alpha Omegan. 1977;70(3):62-65.
8. Hempton TJ, Dominici JT. Contemporary crown-lengthening therapy: A review. JADA 2010;141(6):647-655.

9. Dolt AH 3rd, Robins JW. Altered passive eruption: an etiology of short clinical crowns. Quintessence Int. 1997;28(6):363-72.

10. Escudero-Castaño N, García-García V, Bascones-Llundain J, BasconesMartínez A. Alargamiento coronario, una necesidad de retención protésica, estética y anchura biológica Revisión bibliográfica. Av Odontoestomatol. 2010;23(4):171180.

11. Joly JC, Mesquita CPF, Carvalho SR. Flapless aesthetic crown lengthening: A new therapeutic approach. Rev Mex Periodontol. 2011;2(3):103-108.

12. Nethravathy R, Vinoth SK, Thomas AV. Three different surgical techniques of crown lengthening: A comparative study. J Pharm Bioall Sci. 2013;5:14-6.

13. Stevens BH, Levine RA. Forced eruption: a multidisciplinary approach for form, function, and biologic predictability. Compend Contin Educ Dent. 1998 19(10):994-8. 